EDITORIAL

Special Feature "Meta-analysis in plant ecology"

Running headline: Advancing plant ecology through meta-analyses

\title{
Advancing plant ecology through meta-analyses
}

Lorena Gómez-Aparicio $^{1^{*}}$ and Christopher J. Lortie ${ }^{2}$

${ }^{1}$ Instituto de Recursos Naturales y Agrobiología (IRNAS), CSIC, PO Box 1052, Sevilla 41080, Spain

${ }^{2}$ York University, Department of Biology, Toronto, ON M3J 1P3, Canada.

*Correspondence author. E-mail address: lorenag@irnase.csic.es 


\section{Summary}

2 1. The inherent complexity of nature produces a diverse and varied set of outcomes for

3 any given ecological process. However, the advance of ecology requires making

4 generalizations that synthesize current knowledge and guide new basic research and

5 practical applications. Amongst the synthesis tools available for this specific purpose,

6 meta-analysis is one of the most accurate and powerful methods.

7 2. This Special Feature examines the use that meta-analysis has received in plant

8 ecology over the last two decades and provides examples of synthesis applied to

9 contemporary topics in different areas of plant ecology from populations to ecosystems.

10 3. The number of meta-analyses in plant ecology has been increasing rapidly in the last

11 two decades. However, this increase has not been accompanied by a parallel increase in quality. The opening review paper in this Special Feature provides a checklist of quality criteria specific to ecological meta-analysis that will largely contribute to improvement of the methodological and reporting standards of meta-analyses.

4. The following five papers in the Special Feature demonstrate the advantages of application of meta-analysis compared to other techniques of research synthesis. Metaanalysis is applied here to demonstrate the consistency of ecological hypotheses across large spatial scales (e.g. Janzen-Connell hypothesis), understand sources of variation in the magnitude of ecological processes (e.g. herbivory effects on leaf life span, effects of intraspecific genetic diversity on communities and ecosystems), measure synergistic impacts of environmental change drivers (e.g. $\mathrm{CO}_{2}$, drought, land-use) or assess research gaps within a certain sub-discipline of plant ecology (e.g. landscape fragmentation).

5. Synthesis Meta-analysis can contribute to the advance of ecological theory by synthesizing the available evidence on specific topics and informing the scope of 
generalizations. However, plant ecologists can only take full advantage of this capacity if we improve our knowledge on how and when to conduct a proper meta-analysis, and by avoiding the frequent misuses that have characterized the use of this statistical tool in the ecological literature thus far.

Key-words: data synthesis, effect sizes, global change drivers, grassland function, intraspecific genetic diversity, Janzen-Connell hypothesis, landscape fragmentation, plant-herbivore interactions, plant population and community dynamics, strength of evidence

\section{Introduction}

Probably all scientists have at one time felt overwhelmed by the abundance of literature on any particular research topic, the finding of contradictory results, and the prevalence of apparently idiosyncratic patterns. Because of the inherent complexity of nature, it is probably true that, for any given ecological process, a wide set of outcomes can be found under particular combinations of species and environmental factors. However, the science of ecology can only advance if we are able to discern the exception from the rule, making generalizations that synthesize current knowledge and guide new basic research and practical applications. This fact is particularly true under a global change scenario, where ecologists are pressured to provide accurate quantitative assessments for the effects of major environmental drivers and facilitate evidence-based decision making. The field of research synthesis is devoted to the integration of findings of primary research. Research synthesis will provide the means to evaluate the evidence for alternative hypotheses and examine generalizations in any discipline including ecology if transparently described (Lortie et al. 2014). Meta-analysis is one of the many 
51 tools available but also likely the most direct technique in many respects in summarizing evidence for a particular topic (Arnqvist \& Wooster 1995, Nakagawa \& Poulin 2012, Koricheva \& Gurevitch 2013). Meta-analysis is a set of statistical methods for combining the magnitude of the outcomes (effect sizes) across different data sets addressing the same research question (sensu Koricheva \& Gurevitch). Meta-analyses therefore provide an assessment of the strength of evidence of the respective primary research, and this is critical for decision makers. Moreover, derived datasets and replicable syntheses will advance the state of knowledge for plant ecologists and will provide the capacity to identify limitations and research gaps in the literature.

This Journal of Ecology Special Feature aims to examine the use that metaanalysis has received in plant ecology over the last two decades and provide examples of synthesis successfully applied to provide new insights into contemporary topics in plant ecology. The Special Issue consists of 6 papers. The opening paper by Koricheva \& Gurevitch (2014) offers the first review of the applications and contribution of metaanalysis to plant ecology and assesses the methodological and reporting quality of metaanalysis in this field. Koricheva \& Gurevitch (2014) show that the number of metaanalyses in plant ecology has increased substantially during the last 20 years. This increase has been fostered by the accumulation of a sufficient number of individual studies to make scientists consider that the time was ripe for meta-analysis as well as by the popularization of meta-analytical statistical techniques. Whereas in the past, a large majority of the meta-analyses published in ecological journals used the software MetaWin (Rosenberg et al. 2000), today there is a much wider variety of options due to the development of new and complete open-access software such as $\mathrm{R}$ packages metahdep (Stevens \& Taylor 2009), metafor (Viechtabuer 2010), MCMCglmm (Hadfield 2010), and phyloMeta (Lajeunesse 2011), or the more recent OpenMEE 
(Dietz et al. 2013). This availability opens a whole world of possibilities for conducting meta-analyses of very different degrees of sophistication from the classic meta-analysis with a frequentist approach (e.g. Zvereva \& Kozlov 2014, Thebault et al. 2014) to the most novel Bayesian meta-analysis (e.g. Kulmatiski et al. 2008, Whitlock 2014, Ibáñez et al. 2014). The diversity of options is reflected in the five studies that accompany the review by Koricheva \& Gurevitch in this Special Feature.

Despite the increase in the number of papers, a worrisome conclusion of the Koricheva \& Gurevitch review is the fact that we have not gained enough in quality over time. These authors highlighted the surprisingly high number of studies where the term “meta-analysis” was used but accepted meta-analysis methodology was not present in the synthesis (see Coté \& Reynolds 2012 and Vetter et al. 2013 for similar conclusions in related sub-disciplines). For example, meta-analysis is still confounded with vote-counting or used anytime data from several studies are extracted and analyzed in some way. The original definition of meta-analysis, coined by Glass in 1976 in the field of educational science, was "the statistical analysis of a large collection of results from individual studies for the purpose of integrating findings". This was probably too general and could have led to the current misuse of the term. At present however, ecological meta-analysis has a much more precise definition, i.e., it must include effect size estimates (Koricheva \& Gurevitch 2013, Lortie et al. 2014). Conducting a metaanalysis therefore involves a well-defined number of steps and associated methods that have been summarized in the first handbook of meta-analysis written specifically for ecologists and evolutionary biologists (Koricheva et al. 2013). In an additional effort to raise the standards of meta-analysis in plant ecology, Koricheva \& Gurevitch (2014) provide for the first time a checklist of quality criteria specifically for ecological metaanalysis that compile and improve previous lists proposed for assessing the quality of 
meta-analysis in ecology and related areas (Philibert et al. 2012, Vetter et al. 2013). We are confident that this checklist will contribute to a consistent and well-defined usage of the term meta-analysis by plant ecologists, to improve the reporting standards of future meta-analyses, and to help reviewers and editors identify meta-analyses that do not meet basic quality pre-requisites.

Meta-analysis has clear advantages over other qualitative and quantitative techniques of research synthesis. First, meta-analysis allows for an estimate of the magnitude (not only the existence) of an effect across studies thereby taking into account the sample size and statistical accuracy of the individual studies combined. Second, meta-analysis can be applied to compare the magnitude of an effect on different related response variables (e.g. different components of landscape fragmentation, Ibáñez et al. 2014) offering a comprehensive assessment of the effects of complex processes on plants. Third, because it allows using covariates (i.e. explanatory factors) to understand sources of variation in the magnitude of the effect, it contributes to the clarification of the circumstances associated with increased likelihoods of positive effects. In fact, it is not uncommon to find meta-analyses wherein the overall effect size is small or nonsignificant but comparisons of aggregated sets of effect sizes are significant such as groups of species, sites, or ecological conditions (e.g. Knorr et al. 2005, GómezAparicio 2009). The studies included in this Special Feature have been selected to illustrate these advantages for very different areas of plant ecology from populations to ecosystems.

\section{Advancing plant population ecology through meta-analysis}

Meta-analysis has a very real capacity to contribute to theory development in plant ecology. In the second paper of this Special Feature, Comita et al. (2014) present an 
excellent example of this opportunity by providing an updated, thorough synthesis of one of the most influential hypotheses in plant ecology, the Janzen-Connell hypothesis (Janzen 1970; Connell 1971). In contrast with the only meta-analysis conducted on the topic to date (Hyatt et al. 2003), Comita et al. (2014) found significant support for both the distance- and density-dependent predictions of the Janzen-Connell hypothesis. Differences among both meta-analyses are explained on the basis of differences in the number of studies included (higher in Comita et al. 2014) as well as on a suite of fundamental decisions regarding study selection and integration into the meta-analysis. The work by Comita et al. nicely illustrates the advantages offered by meta-analysis to explore the consistency of an ecological hypothesis across large spatial scales difficult to cover in single studies. They compared the weight of evidence for the predictions of the Janzen-Connell hypothesis across latitudinal and precipitation gradients and by ecosystem types (temperate vs. tropical) and continents. There was a trend for stronger distance- and density-dependence in wetter sites compared to sites with lower annual precipitation. Comita et al. concluded that their synthesis supports the existence of significant overall effects of conspecific density- and distance-dependence on survival in plant communities worldwide, but that further studies are needed in order to attribute these patterns to natural enemies as suggested by Janzen and Connell.

In the third study of this Special Feature, Whitlock (2014) synthesizes the research evidence around a hot topic, the role of intraspecific genetic diversity for communities and ecosystems. Whereas extensive efforts have focused on understanding and synthesizing species diversity effects on ecosystem function (Balvanera et al. 2006, Cardinale et al. 2006), the role of the intraspecific variation in diversity has been underexplored, and it was also identified as one of the 100 fundamental questions that can guide ecological research in the future (Sutherland et al. 2012). Whitlock (2014) 
151 detected an overall positive effect of population-level adaptive genetic diversity (but not

152

of neutral genetic diversity) on community- and ecosystem-level ecological responses demonstrating that these two measures of intraspecific variation should not be used as ecologically equivalent. Moreover, he found strong variation in the effect of adaptive genetic diversity depending on the community (e.g. richness, evenness) and ecosystem measure (e.g., stocks, fluxes) chosen, as well as on the particular characteristics of the individual studies (e.g. spatial extent of the sampling unit, types of genetic diversity recorded). This synthesis advances the debate about whether relationships between genetic diversity and ecological structure are either positive or negative by showing how the strength and direction of these relationships changes with the different measures of diversity and importantly in different ecological contexts.

\section{Advancing community and ecosystem ecology through meta-analysis}

Meta-analysis has also contributed extensively to explicate the organization and interaction amongst species in plant communities. In fact, the first paper that introduced the methods of meta-analysis in ecology in the early 1990s synthesized the findings at that time for field-competition experiments at different trophic levels (Gurevitch et al. 1992). Since then, meta-analysis has been frequently applied to summarize the variability in the sign and magnitude of plant-plant interactions (Goldberg et al. 1999, Maestre et al. 2005, Lortie \& Callaway 2006, Gómez-Aparicio 2009, He et al. 2013, Liczner \& Lortie 2014), as well as to synthesize research on the interaction among plants and other trophic levels including herbivores (Hawkes \& Sullivan 2001, Stiling \& Cornelissen 2007), animal mutualists (Vázquez et al. 2008) or mycorrhizal fungi (Karst et al. 2008, Hoeksema et al. 2010). In the fourth paper of this Special Feature, Zvereva \& Kozlov (2014) provide a novel contribution to the understanding of plant-herbivore 
interactions by synthesizing for the first time knowledge on the effects of herbivores on leaf life span. They demonstrated an overall negative effect of herbivory on leaf life span suggesting that premature abscission of damaged leaves can be viewed as one of the general responses of woody plants to herbivory. But as for Whitlock (2014), probably the most interesting contribution of this paper was to explain the high variation in such effect as a function of particular characteristics of the independent studies. Specifically, they showed that the variability in plant responses to herbivory depend substantially on species-specific leaf traits, suggesting it might be linked with the leaf economics spectrum (Reich et al. 1999, Wright et al. 2004).

One of the most popular uses of ecological meta-analysis in the last two decades has been the synthesis of independent studies assessing the effects of different global change drivers on plants (Koricheva \& Gurevitch 2014). The last two papers of this Special Feature represent well-executed examples of this application. Ibáñez et al. (2014) presented the most comprehensive analysis to date of the integrated effects of landscape fragmentation on plant species and communities. Interestingly, whereas fragmentation is usually perceived to negatively affect plant communities, they found that both positive and negative responses to fragmentation are common but neither are dominant. None of the covariables used in their analyses (biomes, vegetation types, functional groups, life stages) satisfactorily explained the high heterogeneity of responses found. They conclude that broad generalizations about the effects of fragmentation on plant communities might not be possible due to the large variety of processes and responses associated with fragmentation. This is a significant conclusion from a synthesis perspective because it clearly identifies the need for additional, context specific research and management. Given this lack of consistent findings, Ibáñez et al. provide a suite of specific recommendations on possible avenues to overcome the 
201 difficulties inherent in the assessment of the effects of the different components of 202 landscape fragmentation (isolation, edge effects, fragment size, time since 203 fragmentation) on plants. This is also an excellent synthesis as it clearly illustrates the power and consistency of Bayesian statistics. Sensitivity analyses are critical in any set of analyses, and given the level of abstraction sometimes needed in meta-analyses, this work provides a useful example for ecological synthesists that tackle highly contextdependent topics or embrace powerful but sometimes challenging analytical tools. Finally, Thebault et al. (2014) used meta-analysis to explore the relative importance of local management practices (fertilization, fire, abandonment, mowing) vs. climate change drivers (increased $\mathrm{CO}_{2}$, warming, drought, flooding) on plant 211 productivity and soil processes. This synthesis provides an example of the use of absolute value contrasts of effect sizes, decoupled from the sign of respective and aggregated effects, as a means to assess the relative importance of drivers between different groups or levels. By contrasting the effect sizes of these different factors, they found that combinations of local management practices had a much larger effect on grassland functions than individual or even the interactive effects of climate change factors. This synthesis thus provides much needed insights into the potential projected responses of grassland ecosystems to climate change because it suggests that localscale, land management practices must be included in global models. Moreover, this work emphasizes a frequently assumed, but rarely demonstrated ecological principle, that the synergistic impacts of several drivers of environmental change have greater effects on plant communities and ecosystems than any one factor acting in isolation.

\section{Conclusions}


225 The studies included in this Special Feature demonstrate that meta-analysis has the capacity to contribute to the advance of ecological theory by synthesizing the available

227 evidence on specific topics and informing the scope of broad generalizations. Independently of whether the conclusion of the studies was the existence (e.g. Comita et al. 2014) or not (e.g. Ibáñez et al. 2014) of enough evidence in support of the specific hypothesis tested, all syntheses included here identified the gaps of knowledge needed to inform future research efforts by avoiding replicated studies and inspiring novel approaches. The significant benefits that the application of meta-analytical techniques can provide are however limited to some extent by their frequent misuse in plant ecology (Koricheva \& Gurevitch 2014). We still lag behind other disciplines such as medicine or the social sciences in the correct or effective use of meta-analysis (Roberts et al. 2006, Lau et al. 2013). Differences in decisions regarding the number and type of studies included in a meta-analysis or in the degree of conservatism of the statistical techniques applied can change the results of meta-analyses conducted on a same topic, as shown by two papers in this Special Feature (Comita et al. 2014, Whitlock 2014). To ensure that meta-ecological analyses illuminate and summarize effectively, we need to improve our knowledge on how and when to conduct a proper meta-analysis and also take advantage of available cutting-edge meta-analytical methods (e.g. Curtis \& Queenborough 2012). We hope that this Special Feature serves as a useful starting point for these efforts.

\section{Acknowledgements}

We thank the editorial team of Journal of Ecology for encouraging and supporting the development of this special feature and all participating authors for their highly 
interesting contributions. L.G.A. would also like to acknowledge the support from the Spanish Ministry of Science and Innovation (project CGL2011-26877).

\section{References}

Arnqvist, G. \& Wooster, D. (1995) Meta-analysis: synthesizing research findings in ecology and evolution. Trends in Ecology and Evolution, 10, 236-240.

Balvanera, P., Pfisterer, A. B., Buchmann, N., He, J-S., Nakashizuka, T., Raffaelli. D. \& Schmid. B. (2006) Quantifying the evidence for biodiversity effects on ecosystem functioning and services. Ecology Letters, 9, 1146-1156.

Cardinale, B. J., Srivastava, D. S., Duffy, J. E., Wright, J. P, Downing, A. L., Sankaran, M., \& Jouseau, C. (2006) Effects of biodiversity on the functioning of trophic groups and ecosystems. Nature, 443, 989-992.

Comita, L., Queenborough, S., Murphy, S., Eck, J., Xu, K., Krishnadas, M. et al. (2014) Testing predictions of the Janzen-Connell hypothesis: A meta-analysis of experimental evidence for distance- and density-dependent seed and seedling survival. Journal of Ecology, X, 00-00.

Connell, J. H. (1971) On the role of natural enemies in preventing competitive exclusion in some marine animals and in rain forest trees. Dynamics of Populations (eds P. J. den Boer \& G. R. Gradwell), pp. 298-312. Centre for Agricultural Publishing and Documentation, Wageningen, The Netherlands.

Coté, I. M. \& Reynolds, J. D. (2012) Meta-analysis at the intersection of evolutionary ecology and conservation. Evolutionary Ecology, 26, 1237-1252.

Curtis, P. S. \& Queenborough, S. A. (2012) Raising the standards for ecological metaanalyses. New Phytologist, 195, 279-281.

Dietz, G., Dahabreh, I. J., Trikalinos, T. A., Schmid, C. H., Gurevitch, J., Lajeunesse, 
M. J. et al. (2013) OpenMEE: Software for Ecological and Evolutionary MetaAnalysis. Available at: http://www.cebm.brown.edu/open_mee.

Glass, G.V. (1976) Primary, secondary, and meta-analysis of research. Educational Researcher, 5, 3-8.

Goldberg, D.E., Rajaniemi, T., Gurevitch, J. \& Stewart-Oaten, A. (1999) Empirical approaches to quantifying interaction intensity: competition and facilitation along productivity gradients. Ecology, 80, 1118-1131.

Gómez-Aparicio, L. (2009) The role of plant interactions in the restoration of degraded ecosystems: a meta-analysis across life-forms and ecosystems. Journal of Ecology, 97, 1202-1214.

Gurevitch, J., Morrow, L.L., Wallace, A. \& Walsh, J.S. (1992) A meta-analysis of competition in field experiments. American Naturalist, 140, 539-572.

Hadfield, J. D. (2010) MCMC methods for multi-response generalised linear mixed models: the MCMCglmm R package. Journal of Statistical Software, 33, 1-22.

Hawkes, C.V. \& Sullivan, J.J. (2001) The impact of herbivory on plants in different resource conditions: a meta-analysis. Ecology, 82, 2045-2058.

He, Q., Bertness, M.D., \& Altieri, A.H. (2013) Global shifts towards positive species interactions with increasing environmental stress. Ecology Letters, 16, 695-706.

Hoeksema, J.D., Chaudhary, V.B., Gehring, C.A., Johnson, N.C., Karst, J., Koide, R.T. et al (2010) A meta-analysis of context-dependency in plant response to inoculation with mycorrhizal fungi. Ecology Letters, 13, 394-407.

Hyatt, L. A., Rosenberg, M. S., Howard, T. G., Bole, G., Fang, W., Anastasia, J. et al. (2003) The distance dependence prediction of the Janzen-Connell hypothesis: a meta-analysis. Oikos, 103, 590-602. 
Ibáñez, I., Katz, D., Peltier, D., Wolf, S. \& Connor Barrie, B. (2014) Assessing integrated effects of landscape fragmentation on plants and plant communities: the challenge of multiprocess-multiresponse dynamics. Journal of Ecology, X, 00-00.

Janzen, D. H. (1970) Herbivores and the number of tree species in tropical forests. The American Naturalist, 104, 501-528.

Karst, J., Marczak, L., Jones, M.D. \& Turkington, R. (2008) The mutualism-parasitism continuum in ectomycorrhizas: a quantitative assessment using meta-analysis. Ecology, 89, 1032-1042.

Knorr, M., Frey, S. D. \& Curtis P. S. (2005) Nitrogen additions and litter decomposition: a meta-analysis. Ecology, 86, 3252-3257.

Koricheva, J., Gurevitch, J. \& Mengersen, K. (eds.) (2013) Handbook of meta-analysis in ecology and evolution. Princeton University Press, Princeton and Oxford.

Koricheva, J. \& Gurevitch, J. (2013) Place of meta-analysis among other methods of research synthesis. Pp 3-13 in Koricheva J., Gurevitch, J. \& Mengersen, K. (eds) Handbook of meta-analysis in ecology and evolution. Princeton University Press, Princeton and Oxford.

Koricheva, J. \& Gurevitch, J. (2014) Use and misuse of meta-analysis in plant ecology. Journal of Ecology, X, 00-00.

Kulmatiski, A., Beard, K. H., Stevens, J. R. \& Cobbold, S. M. (2008) Plant-soil feedbacks: a meta-analytical review. Ecology Letters, 11, 980-992.

Lajeunesse, M. J. (2011) phyloMeta: a program for phylogenetic comparative analyses with meta-analysis. Bioinformatics, 27, 2603-2604.

Liczner, A.R. \& Lortie, C.J. (2014) A global meta-analytic contrast of cushion-plant effects on plants and on arthropods. PeerJ, 2, e265.

Lortie, C.J., Stewart, G., Rothstein, H., \& J., L. (2014) How to critically read ecological meta- 
analyses. Research Synthesis Methods. http://dx.doi.org/10.1002/jrsm.1109

Lortie, C.J. \& Callaway, R.M. (2006) Re-analysis of meta-analysis: support for the stress-gradient hypothesis. Journal of Ecology, 94, 7-16.

Maestre, F.T., Valladares, F. \& Reynolds, J.F. (2005) Is the change of plant-plant interactions with abiotic stress predictable? A meta-analysis of field results in arid environments. Journal of Ecology, 93, 748-757.

Nakagawa, S. \& Poulin, R. (2012) Meta-analytical insights into evolutionary ecology: an introduction and synthesis. Evolutionary Ecology, 26, 1085-1099.

Philibert, A., Loyce, C. \& Makowski, D. (2012) Assessment of the quality of metaanalysis in agronomy. Agriculture, Ecosystems and Environment, 148, 72-82.

Reich, P.B., Ellsworth, D.S., Walters, M.B., Vose, J.M., Gresham, C., Volin, J.C. et al (1999) Generality of leaf trait relationships: a test across six biomes. Ecology, 80, 1955-1969.

Roberts, P. D., Stewart, G. B. \& Pullin, A. S. (2006) Are review articles a reliable source of evidence to support conservation and environmental management? A comparison with medicine. Biological Conservation, 132, 409-423.

Rosenberg, M. S., Adams, D. C. \& Gurevitch, J. (2000) MetaWin: statistical software for meta-Analysis, Version 2. Sinauer Associates, Sunderland.

Sutherland, W. J., Freckleton, R. P., Godfray, H. C. J., Beissinger, S. R., Benton, T., Cameron, D. D. et al. (2012) Identification of 100 fundamental ecological questions. Journal of Ecology, 101, 58-67.

Stevens, J. R. \& Taylor, A. M. (2009) Hierarchical dependence in meta-analysis. Journal of Educational and Behavioral Statistics, 34, 46-73.

Stiling, P. \& Cornelissen T. (2007) How does elevated carbon dioxide (CO2) affect plant-herbivore interactions? A field experiment and meta-analysis of CO2-mediated 
EDITORIAL

changes on plant chemistry and herbivore performance. Global Change Biology, 13, 1823-1842.

350

Thébault, A., Mariotte, P., Lortie, C. J. \& MacDougall, A. (2014) Local management practices trump the effects of climate change and elevated $\mathrm{CO}_{2}$ on grassland functioning. Journal of Ecology, X, 00-00.

Vazquez, D.P., Morrris, W.F. \& Jordano, P. (2005) Interaction frequency as a surrogate for the total effect of animal mutualists on plants. Ecology Letters, 8, 1088-1094.

Vetter, D., Rücker, G. \& Storch, I. (2013) Meta-analysis: a need for well-defined usage in ecology and conservation biology. Ecosphere, 4, 74.

Viechtbauer, W. (2010) Conducting meta-analyses in R with the metafor package. Journal of Statistical Software, 36, 1-48.

Whitlock, R. (2014) Relationships between adaptive and neutral genetic diversity and ecological structure and function: a meta-analysis. Journal of Ecology, X, 00-00.

Wright, I.J., Reich, P.B., Westoby, M., Ackerly, D.D., Baruch, Z., Bongers, F. et al. (2004) The worldwide leaf economics spectrum. Nature, 428, 821-827.

Zvereva, E. \& Kozlov, M. (2014) Effects of herbivory on leaf life span in woody plants: a meta-analysis. Journal of Ecology, X, 00-00. 